\title{
OPEN Local environment effects on charged mutations for developing aggregation-resistant monoclonal antibodies
}

\begin{abstract}
Jihyeon Lee, Song-Ho Chong \& Sihyun Ham $₫$
Protein aggregation is a major concern in biotherapeutic applications of monoclonal antibodies. Introducing charged mutations is among the promising strategies to improve aggregation resistance. However, the impact of such mutations on solubilizing activity depends largely on the inserting location, whose mechanism is still not well understood. Here, we address this issue from a solvation viewpoint, and this is done by analyzing how the change in solvation free energy upon charged mutation is composed of individual contributions from constituent residues. To this end, we perform molecular dynamics simulations for a number of antibody mutants and carry out the residue-wise decomposition of the solvation free energy. We find that, in addition to the previously identified "global" principle emphasizing the key role played by the protein total net charge, a local net charge within $\sim 15 \AA$ from the mutation site exerts significant effects. For example, when the net charge of an antibody is positive, the global principle states that introducing a positively charged mutation will lead to more favorable solvation. Our finding further adds that an even more optimal mutation can be done at the site around which more positively charged residues and fewer negatively charged residues are present. Such a "local" design principle accounts for the location dependence of charged mutations, and will be useful in producing aggregation-resistant antibodies.
\end{abstract}

Monoclonal antibodies are a growing class of biological therapeutics targeting a broad range of human disorders such as cancers and autoimmune diseases ${ }^{1-3}$. However, the development and application of antibodies as biomedicines have been severely limited due to their high propensities to aggregate under concentrated conditions $^{4,5}$. Aggregation propensity is even more salient for fragments-variable heavy $\left(V_{H}\right)$ and light $\left(V_{L}\right)$ domains responsible for binding to antigens or fusions thereof-which are more preferable forms of the antibodybased regents ${ }^{6,7}$. (In the following, both the full-length antibody and the fragment shall be collectively referred to as antibody for brevity.) Several approaches have therefore been proposed to design aggregation-resistant antibodies ${ }^{8,9}$. Mutating surface residues to charged amino acids is among the promising strategies that enhances solubility and, hence, increases resistance to aggregation ${ }^{10-14}$. In this regard, the antibody's net charge was recognized to be a key determinant of optimal charged mutations ${ }^{15,16}$ : inserting positively charged residues was found to be more effective when the net charge is positive, and negatively charged residues take over the role if the net charge is negative. Since such mutations increment the magnitude of net charge, this was explained by the more strengthened electrostatic repulsion between antibody monomers, which in turn disfavors aggregation. On the other hand, the critical importance of the inserting position of charged mutations on the solubilizing activity was also recognized ${ }^{15-17}$, whose mechanism is still not well understood. This indicates the need to better understand the nature of charged mutations.

The impact of charged mutations on solubilizing activity can also be rationalized from a solvation viewpoint. Indeed, the water-induced attraction is often invoked to account for biomolecular self-assembly ${ }^{18,19}$. Such an interaction can be described by the solvation free energy characterizing the affinity for the solvent water ${ }^{20}$. While it is common to apply the concept of hydrophobicity to individual amino acids, one can argue the "overall protein hydrophobicity" in terms of the solvation free energy defined for a whole protein. In fact, we have 

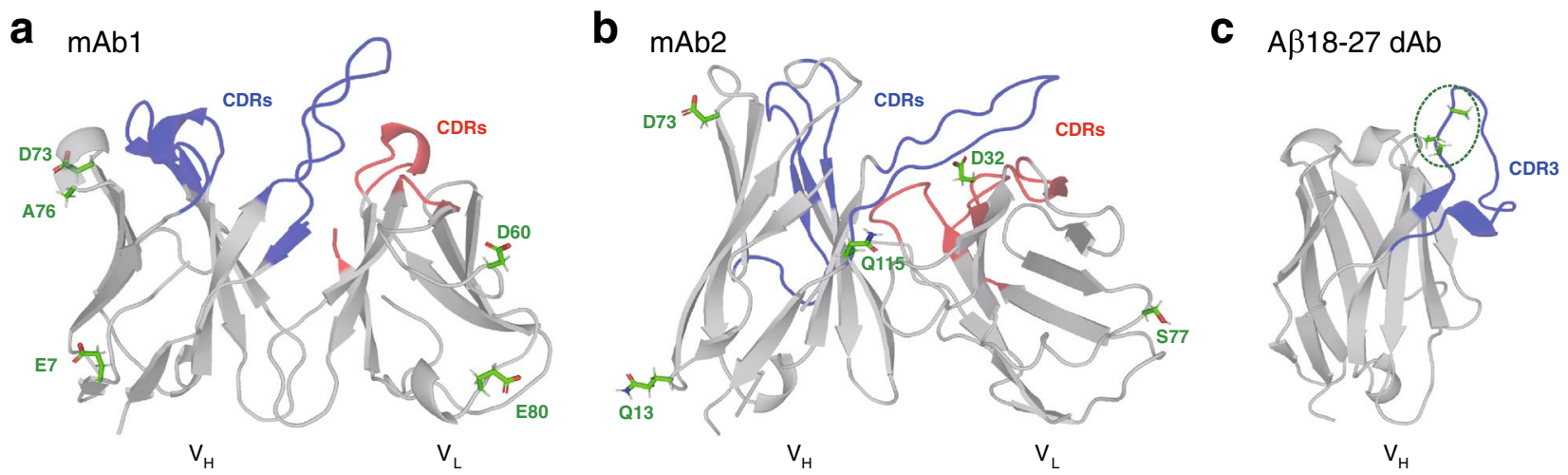

Figure 1. (a, b) Structures of the Fv fragments comprising variable heavy $\left(\mathrm{V}_{\mathrm{H}}\right)$ and light $\left(\mathrm{V}_{\mathrm{L}}\right)$ domains of $(\mathbf{a})$ the wild-type mAb1 (PDB entry 3RU8) and (b) the wild-type mAb2 (modeled based on PDB entry 1DFB). The mutation sites studied in the present work are indicated by stick representations and green labels. Heavy and light complementarity-determining regions (CDRs) are colored blue and red, respectively. (c) Structure of the single-domain $\left(V_{\mathrm{H}}\right)$ antibody $\mathrm{A} \beta 18-27 \mathrm{dAb}$ (modeled based on PDB entry 3B9V). In the "wild type" dAb, three Ala residues (stick representations colored green) are substituted to the mutation sites (enclosed by a green dashed oval) located at the N-terminus of the third CDR (CDR3). PyMOL version 1.8.2 (https://pymol.org) was used to generate protein figures.

recently demonstrated that the overall protein hydrophobicity is the key factor controlling protein aggregation propensity ${ }^{21}$. The same "global" design principle mentioned above-introducing positively (negatively) charged mutations enhances the aggregation resistance when the net charge is positive (negative) - has also been derived from the observation that the solvent water strikingly discriminates positive and negative charges depending on the protein net charge. This solvation perspective was recently adopted by Schäfer and coworkers ${ }^{22}$, who have successfully designed antibody mutants of improved solubility guided by the solvation thermodynamics analysis. On the other hand, it was also found that the variation in solvation free energy upon charged mutation markedly depends on the insertion location, which is outside the scope of the global principle. An additional guiding principle that takes into account the spatial neighbors of the mutation site is therefore necessary.

In this paper, we explore such a "local" design principle for charged mutations from detailed solvation thermodynamics analyses. We investigate the same antibody systems studied in Ref. ${ }^{22}$ since improved solution-state properties of those systems through the optimization of the solvation free energy have been experimentally confirmed. We also analyze the single-domain antibody for which the solubilizing activity of charged mutations was experimentally measured ${ }^{16}$. We conduct molecular dynamics simulations and carry out solvation free energy calculations for these systems. We then perform the residue-wise decomposition analysis of the solvation free energy ${ }^{23-25}$. This allows us to gain detailed knowledge on how the solvation free energy change is composed of individual contributions from constituent residues. We particularly focus on the contributions from the spatial neighbors around the mutation site, whose understanding will provide us with new insights on the insertinglocation dependence of charged mutations. Thereby, we would like to derive a local design principle that will be useful in producing aggregation-resistant antibodies.

\section{Methods}

System description. We investigated the Fv fragments consisting of $\mathrm{V}_{\mathrm{H}}$ and $\mathrm{V}_{\mathrm{L}}$ domains of two monoclonal antibodies (mAbs), to be referred to as mAb1 and mAb2 (Fig. 1a,b). The former (mAb1) is the antibody that neutralizes human immunodeficiency virus-type 1 (HIV-1) $)^{26}$, and the latter (mAb2) is a human anti-DNA autoantibody ${ }^{27}$. The starting structure of mAb1 was taken from the X-ray study (PDB entry $\left.3 \mathrm{RU} 8\right)^{28}$. For mAb2, we built a homology model since no experimental structure is available. This was done with the antibody modeler module in the Molecular Operating Environment (MOE) software ${ }^{29}$ using the structure of PDB entry $1 \mathrm{DFB}^{30}$ as a template. We analyzed 10 mutants of mAb1 and 8 mutants of mAb2 introduced in Ref. ${ }^{22}$ (see Tables 1 and 2). The mutation sites were chosen from the solvent-exposed residues by excluding conserved residues and those within or near the complementarity-determining regions (CDRs; see Fig. 1a,b).

We also studied the single-domain $\left(\mathrm{V}_{\mathrm{H}}\right)$ antibody $(\mathrm{dAb})$, to be referred to as $\mathrm{A} \beta 18-27 \mathrm{dAb}$, which incorporates hydrophobic residues 18-VFFAEDVGSN-27 taken from the Alzheimer's amyloid- $\beta(\mathrm{A} \beta)$ peptide within the third CDR (CDR3; see Fig. 1c). A $\beta 18-27 \mathrm{dAb}$ binds to $\mathrm{A} \beta$ oligomers and fibrils with submicromolar affinity ${ }^{31}$, but it is also prone to self-aggregate within days at $25^{\circ} \mathrm{C}^{16}$. We analyzed 2 mutants of $\mathrm{A} \beta 18-27 \mathrm{dAb}$ in which three charged residues are inserted at the N-terminus of CDR3, whose solubilizing activity was measured experimentally (Table 3$)^{16}$. The starting structure of $\mathrm{A} \beta 18-27 \mathrm{dAb}$ was also modeled with the MOE software ${ }^{29}$ using the structure of $\mathrm{PDB}$ entry $3 \mathrm{~B} 9 \mathrm{~V}^{32}$ as a template.

Molecular dynamics simulations. We used the pmemd.cuda module in AMBER18 package ${ }^{33}$ to perform explicit-water molecular dynamics simulations for all the systems. The ff14SB force field ${ }^{34}$ and TIP3P model ${ }^{35}$ were employed for proteins and water, respectively. The simulations were done under neutral $\mathrm{pH}$, where Glu and 


\begin{tabular}{|c|c|c|c|}
\hline & $\Delta Q^{\mathrm{a}}$ & $\Delta G_{\text {solv }}(\mathrm{kcal} / \mathrm{mol})^{\mathrm{b}}$ & $\Delta G_{\text {solv }}$ from Ref. ${ }^{22}$ \\
\hline \multicolumn{4}{|l|}{ Heavy-chain mutations } \\
\hline E10G & +1 & $-128.2 \pm 0.9$ & $-135.6 \pm 20.5$ \\
\hline $\mathrm{D} 73 \mathrm{~N}$ & +1 & $-85.1 \pm 16.7$ & $-87.4 \pm 19.3$ \\
\hline A76K & +1 & $-121.8 \pm 14.2$ & $-116.1 \pm 21.1$ \\
\hline E10G/D73N/A76K & +3 & $-343.8 \pm 8.4$ & $-396.9 \pm 20.1$ \\
\hline \multicolumn{4}{|l|}{ Light-chain mutations } \\
\hline D60S & +1 & $-73.9 \pm 15.3$ & $-102.7 \pm 21.5$ \\
\hline E80Q & +1 & $-110.4 \pm 4.6$ & $-106.9 \pm 22.5$ \\
\hline D60S/E80Q & +2 & $-160.9 \pm 9.8$ & $-223.7 \pm 21.6$ \\
\hline \multicolumn{4}{|c|}{ Heavy- and light-chain mutations } \\
\hline E10G/A76K/E80Q & +3 & $-268.0 \pm 13.3$ & $-285.7 \pm 21.5$ \\
\hline D73N/A76K/E80Q & +3 & $-282.4 \pm 9.8$ & $-292.3 \pm 22.0$ \\
\hline E10G/D73N/A76K/D60S/E80Q & +5 & $-503.1 \pm 3.5$ & $-484.0 \pm 21.1$ \\
\hline
\end{tabular}

Table 1. Solvation free energy changes upon mutating mAb1. ${ }^{a}$ Increment in charge upon mutation(s); $\mathrm{b} \Delta G_{\text {solv }}=G_{\text {solv }}$ (mutant) $-G_{\text {solv }}$ (wild type).

\begin{tabular}{|c|c|c|c|}
\hline & $\Delta Q^{\mathrm{a}}$ & $\Delta G_{\text {solv }}[\mathrm{kcal} / \mathrm{mol}]^{\mathrm{b}}$ & $\Delta G_{\text {solv }}$ from Ref. ${ }^{22}$ \\
\hline \multicolumn{4}{|l|}{ Heavy-chain mutations } \\
\hline Q13K & +1 & $+12.0 \pm 13.3$ & $-1.5 \pm 13.5$ \\
\hline $\mathrm{D} 73 \mathrm{~N}$ & +1 & $-37.0 \pm 7.0$ & $-82.2 \pm 13.1$ \\
\hline Q115K & +1 & $-43.6 \pm 12.0$ & $-40.2 \pm 13.1$ \\
\hline Q13K/D73N/Q115K & +3 & $-218.1 \pm 11.4$ & $-216.9 \pm 14.1$ \\
\hline \multicolumn{4}{|l|}{ Light-chain mutations } \\
\hline $\mathrm{D} 32 \mathrm{Y}$ & +1 & $+60.2 \pm 19.5$ & $+56.3 \pm 12.5$ \\
\hline S77R & +1 & $+22.8 \pm 11.9$ & $-31.6 \pm 13.8$ \\
\hline $\mathrm{D} 32 \mathrm{Y} / \mathrm{S} 77 \mathrm{R}$ & +2 & $-12.2 \pm 7.1$ & $+10.1 \pm 13.3$ \\
\hline \multicolumn{4}{|l|}{ Heavy- and light-chain mutations } \\
\hline Q13K/D73N/Q115K/D32Y/S77R & +5 & $-316.1 \pm 20.7$ & $-205.6 \pm 13.7$ \\
\hline
\end{tabular}

Table 2. Solvation free energy changes upon mutating mAb2. ancrement in charge upon mutation(s); $\mathrm{b} \Delta G_{\text {solv }}=G_{\text {solv }}($ mutant $)-G_{\text {solv }}($ wild type).

\begin{tabular}{|l|l|l|l|}
\hline & $\boldsymbol{\Delta} \mathbf{Q}^{\mathbf{a}}$ & $\boldsymbol{\Delta} \boldsymbol{G}_{\text {solv }}\left[\mathbf{k c a l} / \mathbf{m o l}^{\mathbf{b}}\right.$ & Aggregation $^{\mathbf{c}}$ \\
\hline RRR-A $\beta 18-27$ & +3 & $-8.5 \pm 4.5$ & Present \\
\hline DDD-A $\beta 18-27$ & -3 & $-507.7 \pm 4.0$ & Eliminated \\
\hline
\end{tabular}

Table 3. Solvation free energy changes upon mutating A $\beta 18-27 \mathrm{dAb}$. ${ }^{\mathrm{a}}$ Increment in charge upon mutation(s);

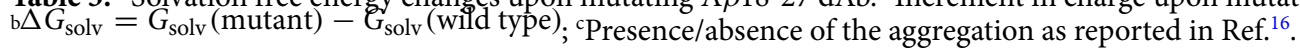

Asp carry a negative charge and Lys and Arg a positive charge. The resulting net charges of $\mathrm{mAb} 1, \mathrm{mAb} 2$ and $\mathrm{A} \beta$ 18-27 dAb are $+6,+3$ and 0 , respectively. The whole charge of each simulation system was neutralized by counter $\mathrm{Cl}^{-}$ions, and additional $\mathrm{Na}^{+}$and $\mathrm{Cl}^{-}$were included to achieve a $150 \mathrm{mM}$ ionic concentration. We applied the particle mesh Ewald method ${ }^{36}$ to handle long-range Coulomb interactions, and short-range interactions were treated by a $10 \AA$ cutoff. Berendsen's thermostat and barostat ${ }^{37}$ were used for constant temperature and pressure (300 K and 1 bar). Two independent 100 ns production simulations were carried out for each system.

Solvation free energy analysis. Based on the general expression for the solvation free energy $G_{\text {solv }}$ known as the Kirkwood charing formula, we have derived the following exact atomic decomposition of $G_{\text {solv }}{ }^{23}$ :

$$
G_{\text {solv }}=\sum_{\alpha} G_{\text {solv }, \alpha}
$$

with 


$$
G_{\text {solv }, \alpha}=4 \pi \sum_{\gamma} \int_{0}^{1} d \lambda \int r^{2} d r \frac{\partial u_{\alpha \gamma}(r ; \lambda)}{\partial \lambda} \rho_{\gamma} g_{\alpha \gamma}(r ; \lambda)
$$

Here $\alpha$ and $\gamma$ refer to the solute (protein) and solvent sites, respectively; $\lambda$ is the coupling parameter that introduces the solute-solvent interaction $u_{\alpha \gamma}(r)$ such that $u_{\alpha \gamma}(r ; \lambda=0)=0$ and $u_{\alpha \gamma}(r ; \lambda=1)=u_{\alpha \gamma}(r) ; \rho_{\gamma}$ is the average number density of site $\gamma$; and $g_{\alpha \gamma}(r ; \lambda)$ is the solute-solvent radial distribution function corresponding to $u_{\alpha \gamma}(r ; \lambda)$. (In the original work ${ }^{23}, \lambda$ consists of two parameters, $\lambda=\left(\lambda_{1}, \lambda_{2}\right)$, to separately control the shortrange and Coulomb interactions, but such a complexity is suppressed here for simplicity.) By an appropriate grouping of contributions from constituent atoms $\left(G_{\text {solv }, \alpha}\right)$, one obtains a residue-wise decomposition of $G_{\text {solv }}$. In the present work, the protein-solvent distribution function was computed from the three-dimensional reference interaction site model (3D-RISM) theory ${ }^{38,39}$ (see the Supplementary Information for details).

\section{Results}

Structural and solvation free energy changes upon mutations in mAb1 and mAb2. We performed explicit-water molecular dynamics simulations for the wild-type and 10 mutants of mAb1 (Table 1) and for the wile-type and 8 mutants of $\mathrm{mAb} 2$ (Table 2). Protein structures were stable during the simulation time (100 ns) in all the systems: the $\mathrm{C}_{\alpha}$ RMSD (root-mean-square deviation) from the respective initial structure stayed within $\sim 3 \AA$. We then computed the solvation free energy $G_{\text {solv }}$ for each system using the simulated structures sampled with a $1 \mathrm{~ns}$ interval. In the following, we will focus on the change in solvation free energy upon mutation defined by $\Delta G_{\text {solv }}=G_{\text {solv }}$ (mutant) $-\mathrm{G}_{\text {solv }}$ (wild type). The average and standard error of $\Delta G_{\text {solv }}$ were estimated based on the two independent production runs. The results for $\mathrm{mAb} 1$ and $\mathrm{mAb} 2$ are presented in Tables 1 and 2, along with the comparison with the previous work. (Results of individual trajectories are reported in Supplementary Table S1 and S2.) Overall, our numerical results for $\Delta G_{\text {solv }}$ are very similar to those reported previously ${ }^{22}$, though some differences are discernible. In particular, $\Delta G_{\text {solv }}$ values for the Q13K, S77R and D32Y/ S77R mutants of mAb2 show the opposite signs compared to the previous work (Table 2). To examine such numerical differences and how they affect the main points (in particular, a local design principle) of the present work, we have carried out two additional independent 100 ns simulations for these systems, and the results are summarized in Supplementary Tables S3 and S4. As demonstrated there, the results from the additional simulations are in better agreement with the previous work, especially concerning the signs of $\Delta G_{\text {solv }}$ values. This indicates that, with two independent $100 \mathrm{~ns}$ simulations, there still remains non-negligible uncertainty in numerical results for $\Delta G_{\text {solv }}$. We will come back to this issue later in Discussion section, where we argue that our main points are nevertheless not significantly altered.

Single-point mutations in mAb1. We start from analyzing the single-point mutants of mAb1. Individual residue contributions to $\Delta G_{\text {solv }}$ upon the E10G (heavy chain) mutation are shown in Fig. 2a; those upon the A76K (heavy chain) mutation in Fig. 2b; and the results for the D73N (heavy chain), D60S (light chain) and E80G (light chain) mutations are displayed in Supplementary Fig. S1. The single-point mutations considered here are either the mutation of a negatively charged residue to a neutral one or the mutation of a neutral residue to a positively charged one, which increments the system charge by +1 . (We recall here that the total charge of the wild-type $\mathrm{mAb} 1$ is +6 .) Hence, according to the aforementioned global principle ${ }^{21}$, positively charged residues get more favorably solvated (resulting in more negative $\Delta G_{\text {solv }}$ ), whereas negatively charged residues become less favorably solvated (more positive $\Delta G_{\text {solv }}$ ). This explains why the contributions to $\Delta G_{\text {solv }}$ from the positively charged residues (colored blue) tend to exhibit negative changes and those from the negatively charged residues (colored red) show the opposite trend.

On the other hand, the presence of charged residues that exhibit more pronounced variations is discernible (indicated by the dashed arrows or SB +/ - in Fig. 2 and Supplementary Fig. S1). We first recognize that such large variations tend to occur in the same chain into which the mutation is introduced: for example, in the heavychain E10G mutation (Fig. 2a), most of the large changes show up in the heavy chain, but not in the light chain. This indicates that the distance to the mutation site matters. We therefore added the distance information $\left(d_{\mathrm{m}}\right.$ in $\AA$; the minimum side-chain heavy atom distance to the mutation site) in Fig. 2 and Supplementary Fig. S1. In fact, we find that the residues exhibiting the large solvation free energy charges are located within $\sim 15 \AA$ from the mutation site. (In this regard, we notice that those distances, and the insets in Fig. 2, were obtained using the wile-type $\mathrm{mAb} 1$ structure. This is because we are interested in recipes for designing aggregation-resistant mutants that can be utilized solely based on the knowledge of the wild-type protein.) Second, we observe a sizable difference in the solvation free energy change at the mutation site (indicated by the green arrow) between the E10G (Fig. 2a) and A76K (Fig. 2b) mutants. This implies that the contents of the mutation (such as a deletion of negatively charged residue versus an addition of positively charged residue) influence $\Delta G_{\text {solv }}$ value at the mutation site. Finally, it is seen that the residues involved in the formation/breaking of salt-bridges (indicated by $\mathrm{SB}+/-$, respectively) upon mutation are significantly affected. However, except for those directly associated with the mutation site ( $\mathrm{SB}+/-$ colored green), it is difficult to predict in advance the formation/breaking of salt-bridges far from the mutation site ( $\mathrm{SB}+/-$ colored black). Therefore, those salt-bridges will not be taken into consideration in our exploration of design principles.

The key to understanding the pronounced variations in $\Delta G_{\text {solv }}$ near and at the mutation site lies in the solvation structure ${ }^{21}$. Since the net charge of $\mathrm{mAb} 1$ is positive $(+6)$, the protein-water electrostatic interaction induces such a long-distance orientational distribution of water where the dipole moment is directed outward from the protein. Those water molecules in turn produce an electrostatic potential that energetically favors positive charges and disfavors negative ones on the protein surface. When a charged mutation of +1 is introduced, such an electrostatic potential strengthens around the mutation site. This explains the large variations in $\Delta G_{\text {solv }}$, 
a $\mathrm{E} 10 \mathrm{G}$ (heavy chain) mutant of $\mathrm{mAb1}\left(\Delta G_{\text {solv }}=-128.2 \mathrm{kcal} / \mathrm{mol}\right)$
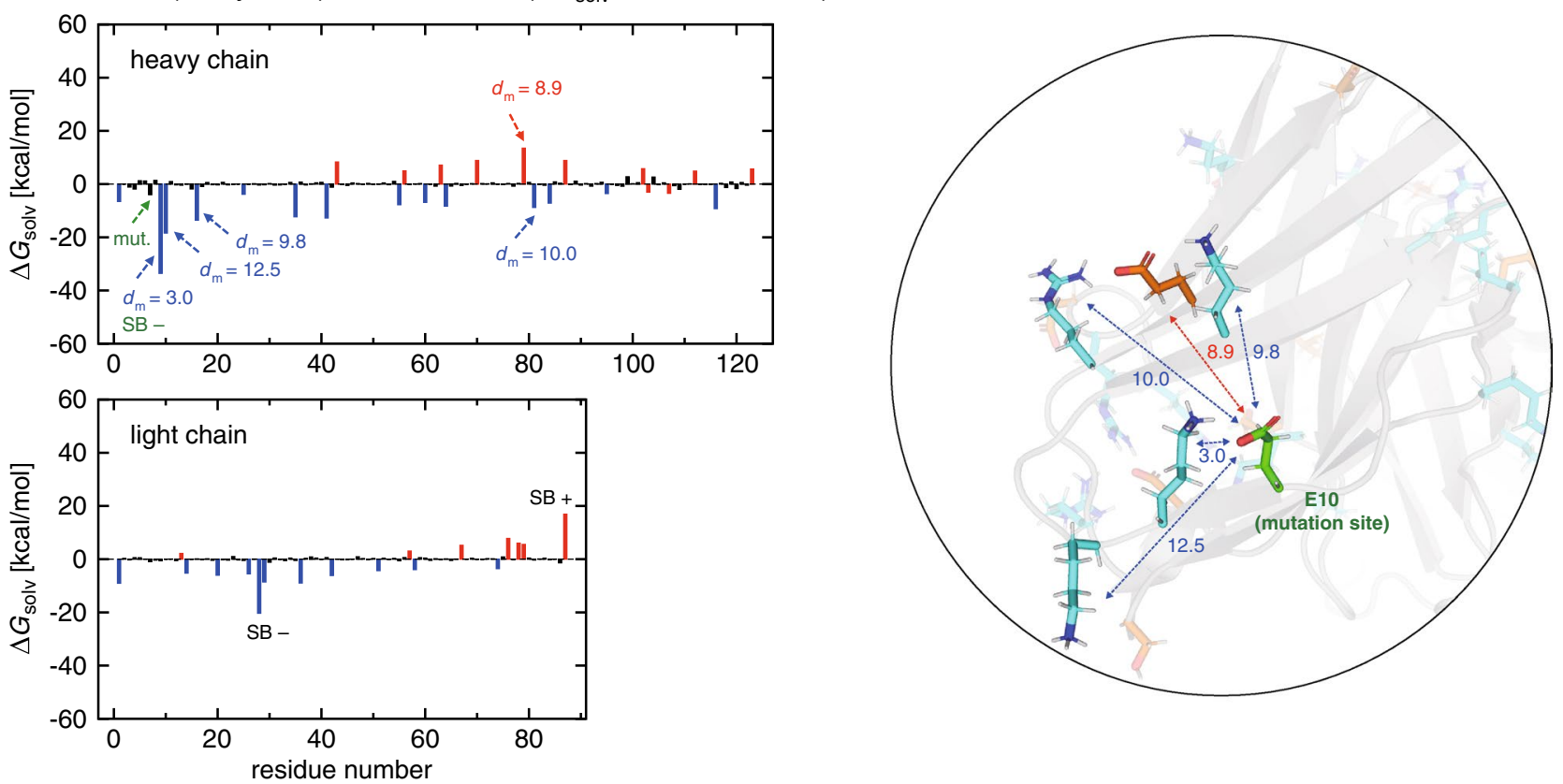

b $\quad \mathrm{A} 76 \mathrm{~K}$ (heavy chain) mutant of $\mathrm{mAb1}\left(\Delta G_{\text {solv }}=-121.8 \mathrm{kcal} / \mathrm{mol}\right)$
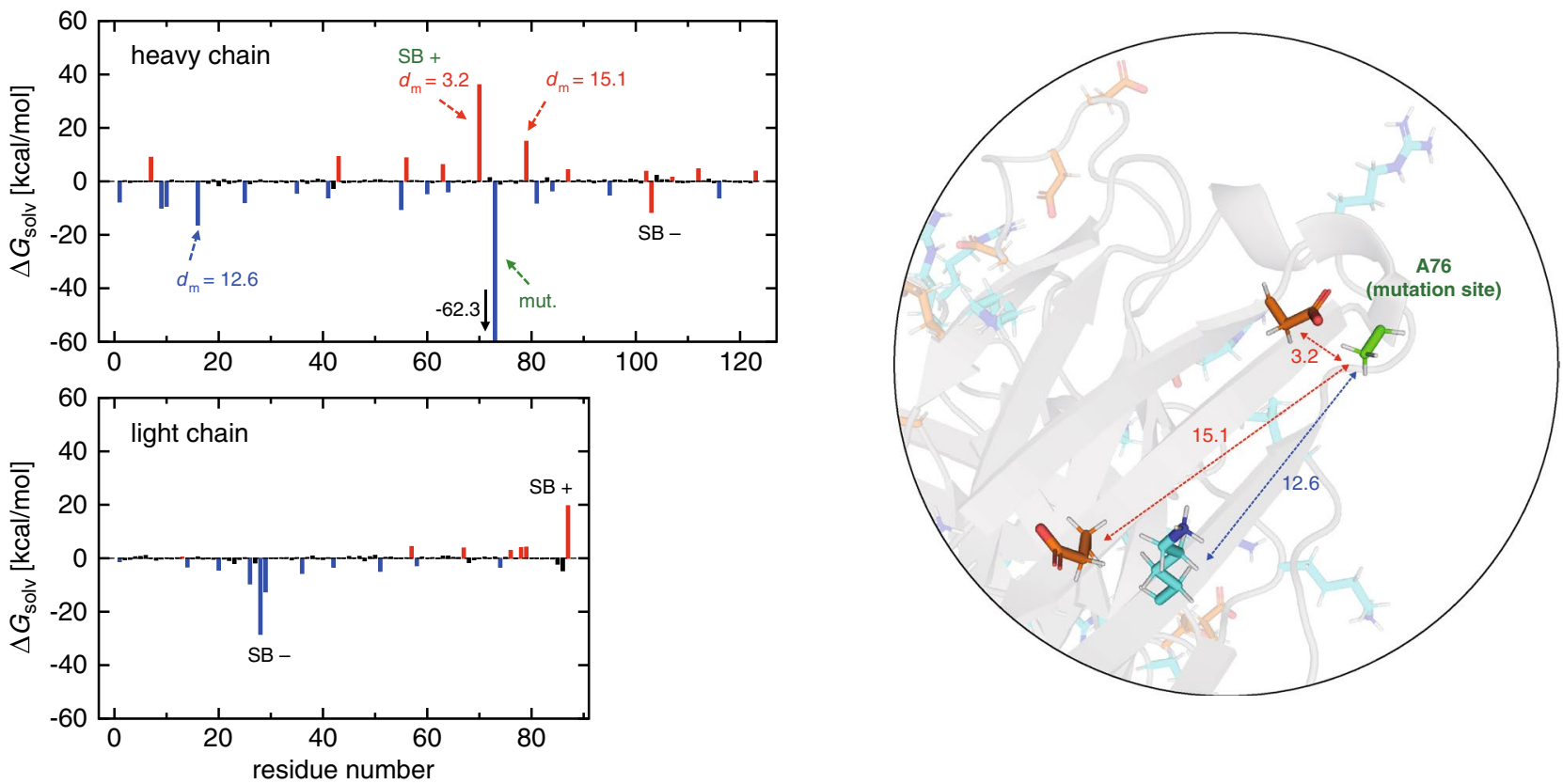

Figure 2. (a, b) Residue-wise decomposition of $\Delta G_{\text {solv }}$ for (a) E10G and (b) A76K mutants of mAb1 (blue, red and black colors refer to positively charged, negatively charged and neutral residues, respectively). The mutation site is indicated by the green arrow. $d_{\mathrm{m}}$ (in $\AA$ ) denotes the distance to the mutation site as illustrated in the inset (green stick representation, mutation site; cyan and orange representations, positively and negatively charged residues, respectively). Residues involved in the formation/breaking of salt-bridges upon mutation are represented by $\mathrm{SB}+/-$ (green if the mutation site is involved, and black otherwise). PyMOL version 1.8.2 (https://pymol.org) was used to generate protein figures.

including their signs, of the residues located within $\sim 15 \AA$ from the mutation site. When this mutation is a deletion of a negatively charged residue, there is no charged residue that is affected by the strengthened electrostatic potential at the mutation site. On the other hand, if the mutation is an addition of a positively charged residue, the mutation site fully appreciates the strengthened electrostatic potential. This accounts for the large difference in $\Delta G_{\text {solv }}$ values at the mutation site between the E10G and A76K mutants. The results for the other mutants shown in Supplementary Fig. S1 can be rationalized in a similar manner. 
a $\quad$ Q13K (heavy chain) mutant of $\mathrm{mAb2}\left(\Delta \mathrm{G}_{\mathrm{solv}}=+12.0 \mathrm{kcal} / \mathrm{mol}\right)$
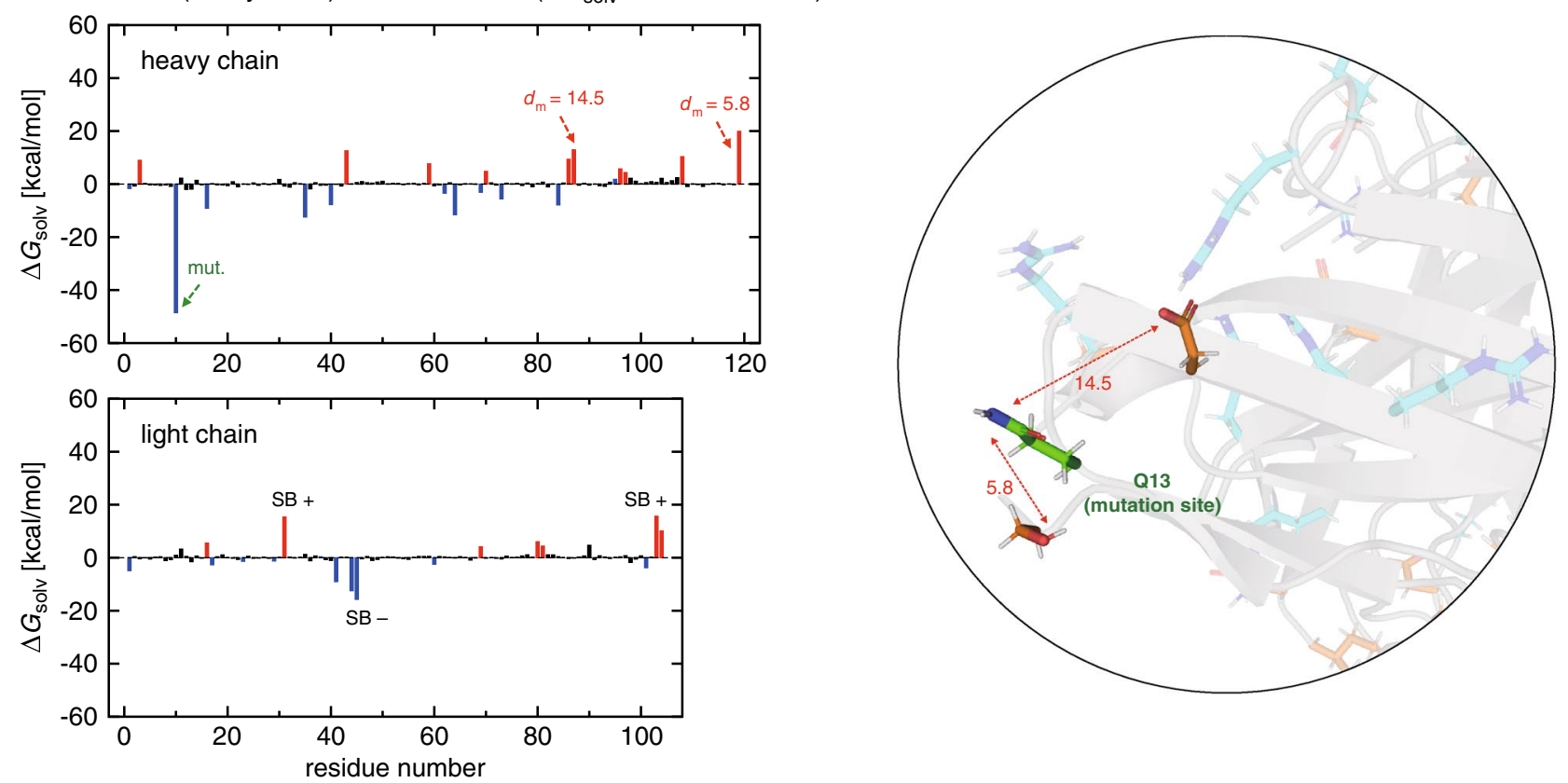

b Q115K (heavy chain) mutant of $\mathrm{mAb2}\left(\Delta G_{\text {solv }}=-43.6 \mathrm{kcal} / \mathrm{mol}\right)$
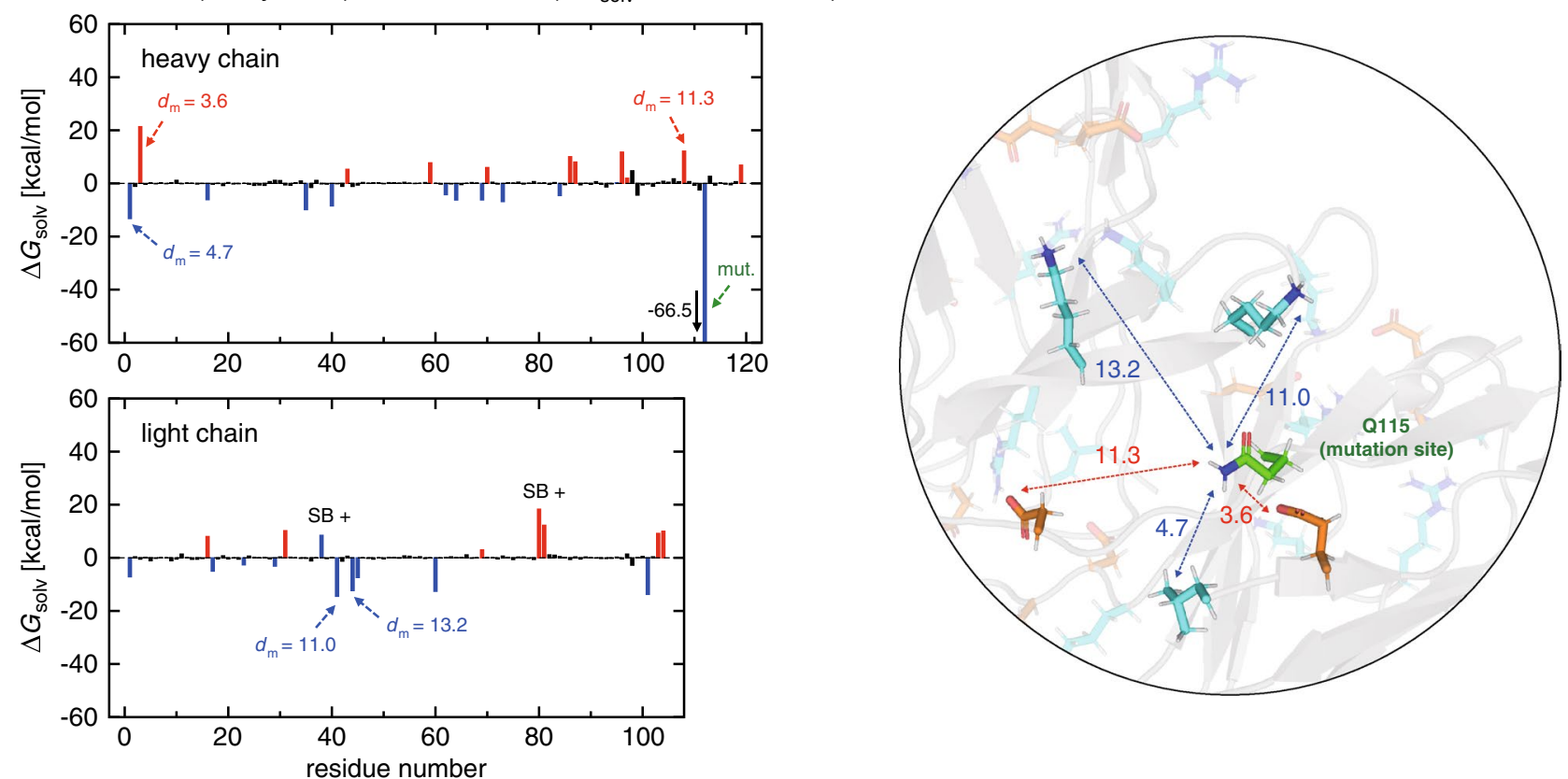

Figure 3. (a, b) Residue-wise decomposition of $\Delta G_{\text {solv }}$ for (a) Q13K and (b) Q115K mutants of mAb2 (blue, red and black colors refer to positively charged, negatively charged and neutral residues, respectively). The mutation site is indicated by the green arrow. $d_{\mathrm{m}}$ (in $\AA$ ) denotes the distance to the mutation site as illustrated in the inset (green stick representation, mutation site; cyan and orange representations, positively and negatively charged residues, respectively). Residues involved in the formation/breaking of salt-bridges upon mutation are represented by $\mathrm{SB}+/-$ (green if the mutation site is involved, and black otherwise). PyMOL version 1.8.2 (https://pymol.org) was used to generate protein figures.

Single-point mutations in mAb2. We next investigate the single-point mutants of mAb2. In contrast to large negative $\Delta G_{\text {solv }}$ values ( -73.9 to $-128.2 \mathrm{kcal} / \mathrm{mol}$; see Table 1) exhibited by the single-point mutants of $\mathrm{mAb} 1$, corresponding $\Delta G_{\text {solv }}$ values of $\mathrm{mAb} 2$ are much less negative (the most negative value is $-43.6 \mathrm{kcal} / \mathrm{mol}$ ) and even take positive values (Table 2). The less negative $\Delta G_{\text {solv }}$ for mAb2 can be understood from the smaller net charge of the wild-type $\mathrm{mAb} 2(+3)$ than that of $\mathrm{mAb} 1(+6)$. Indeed, according to the simple continuum Born model $^{40}$, the solvation free energy change caused by increasing a charge from $Q$ to $Q+\Delta Q(\Delta Q>0)$ is proportional to $-\left(2 Q \Delta Q+\Delta Q^{2}\right)$, and it is negative when $Q>0$ and it becomes more negative for larger $Q$. Thus, 
the positive solvation free energy change upon increasing the net charge observed for the mutants of $\mathrm{mAb} 2$ is counter-intuitive.

To understand such a nontrivial solvation behavior of mAb2, we analyze in detail the Q13K (heavy chain) and Q115K (heavy chain) mutants. These are the same type of mutation (glutamine to lysine), but exhibit strikingly contrasting $\Delta G_{\text {solv }}$ values $(+12.0$ and $-43.6 \mathrm{kcal} / \mathrm{mol}$, respectively) depending on the mutation location, and, hence, constitute good examples for elucidating the position dependence of charged mutations. Residuedecomposed $\Delta G_{\text {solv }}$ values for these mutants are shown in Fig. 3a,b. Main features of Fig. 3a,b can be rationalized in the same way as we explained above for mAb1: since mAb2's net charge is positive, positive (negative) residues tend to exhibit negative (positive) solvation free energy changes; more pronounced effects show up in those residues located within $\sim 15 \AA$ from the mutation site; and the $\Delta G_{\text {solv }}$ value at the mutation site is large negative since a new positively charged residue is introduced there. However, an inspection of Fig. 3a for Q13K and Fig. 3b for Q115K reveals a difference in the distribution of charged residues around $(<\sim 15 \AA)$ the respective mutation site: the Q13K mutation site is surrounded by two negatively charged residues, whereas the Q115K mutation site by two negatively charged residues and three positively charged residues. Those nearby negatively charged residues provide significant positive contributions to $\Delta G_{\text {solv }}$, and this accounts for the positive $\Delta G_{\text {solv }}$ of the Q13K mutant. On the other hand, in the case of Q115K, such large positive contributions to $\Delta G_{\text {solv }}$ arising from the nearby negatively charged residues are sufficiently compensated by large negative contributions from the nearby positively charged residues. Thus, a local net charge within $\sim 15$ A from the mutation site exerts significant impacts on the $\Delta G_{\text {solv }}$ value. This also provides an explanation of the location dependence of charged mutation from the solvation perspective.

Individual residue contributions to $\Delta G_{\text {solv }}$ of the other single-point mutants of mAb2-D73N (heavy chain; $\Delta G_{\text {solv }}=-37.0 \mathrm{kcal} / \mathrm{mol}$ ), D32Y (light chain; $\Delta G_{\text {solv }}=+60.2 \mathrm{kcal} / \mathrm{mol}$ ), and S77R (light chain; $\Delta G_{\text {solv }}=+22.8$ $\mathrm{kcal} / \mathrm{mol}$ ) shown in Supplementary Fig. S2 - can be rationalized in a similar manner. $\Delta G_{\text {solv }}$ for the D73N mutant is largely negative since the mutation site is surrounded by three positively charged residues. $\Delta G_{\text {solv }}$ for the D32Y mutant is positive mainly because a bulky side chain is introduced at the solvent exposed residue, which produces a large positive $\Delta G_{\text {solv }}$ at the mutation site. This suggests that such a mutation that introduces a solvent-exposed bulky side chain should be avoided. $\Delta G_{\text {solv }}$ for the S77R mutant is positive since the mutation site is surrounded by two positively charged residues and three negatively charged residues.

Multipoint mutations in mAb1 and mAb2. Finally, we deal with the multipoint mutations. Because of the larger $(\geq+2)$ increment of the net charge in these mutations, the protein-water electrostatic effects on $\Delta G_{\text {solv }}$ become stronger. Therefore, positive (negative) residues exhibit more pronounced negative (positive) solvation free energy changes than those observed in the single-point mutations. This is seen, e.g., in Fig. 4a showing individual residue contributions to $\Delta G_{\text {solv }}$ of the E10G/D73N/A76K (heavy chain) mutant of mAb1. We also notice from this figure that the solvation free energy changes induced by the three mutations can roughly be described as a superposition of the effects caused by individual mutations: the five residues strongly affected by the single E10G mutation (indicated by the dashed arrows in Fig. 2a) are shown here with the cyan arrows; the three residues associated with the A76K mutation (taken from Fig. 2b) are represented by the magenta arrows; and the three residues whose variations are large in the D73N mutant (Supplementary Fig. S1) are indicated by the orange arrows. (A certain complexity arises here since a residue that exhibits a large solvation free energy change upon one mutation may be deleted in another mutation. Such a case is exemplified by the dashed magenta arrow in Fig. 4a.) The corresponding result for the D60S/E80Q mutations in the light chain of $\mathrm{mAb1}$ (Fig. 4b) can be rationalized likewise in terms of the individual D60S and E80Q mutations shown in Supplementary Fig. S1. The result for the E10G/D73N/A76K/D60S/E80Q mutations (Fig. 4c) can also be roughly described as a superposition of those shown in Fig. 4a,b, and this applies to the other (E10G/A76K/E80Q and D73N/A76K/E80Q) multipoint mutants of mAb1 (Supplementary Fig. S3).

The residue-wise decomposition of $\Delta G_{\text {solv }}$ for the multipoint mutants of $\mathrm{mAb} 2$, shown in Fig. 5, can be understood similarly. The solvation free energy change for the D32Y/S77R mutant $\left(\Delta G_{\text {solv }}=-12.2 \mathrm{kcal} / \mathrm{mol}\right)$ is still not large negative in spite of the double-point charged mutation. This is not only because the bulky side chain (from D32Y) is involved, but also because five positively charged residues and six negatively charged residues are present close to those mutation sites (Fig. 5b). Upon introducing additional charged mutations, more positively charged residues from the Q13K/D73N/Q115K mutations (Fig. 5a) come into play, and the solvation free energy change for the Q13K/D73N/Q115K/D32Y/S77R mutant becomes a large negative value (Fig. 5c).

Multipoint mutations in A $\beta 18-27 \mathbf{d A b}$. Now let us turn our attention to A $\beta 18-27 \mathrm{dAb}$. We studied the RRR-A $\beta 18$-27 and DDD-A $\beta 18-27$ mutants in which three charged residues (RRR or DDD) are inserted at the $\mathrm{N}$-terminus of CDR3 (Fig. 1c). For the computation of $\Delta G_{\text {solv }}$ and its residue-wise decomposition, we also analyzed AAA-A $\beta 18-27$ (i.e., three alanine residues are substituted to the mutation sites) which was considered as the wild-type. We carried out two independent $100 \mathrm{~ns}$ molecular dynamics simulations for these systems, and the simulation structures were stable for all the systems ( $C \alpha$-RMSD stayed within $\sim 3 \AA)$. We then computed the solvation free energy change $\Delta G_{\text {solv }}=G_{\text {solv }}$ (mutant) $-G_{\text {solv }}$ (wild type), and the results are summarized in Table 3 (see also Supplementary Table S5).

The net charge of the wild-type $\mathrm{A} \beta 18-27 \mathrm{dAb}$ is 0 , and its change is symmetrical for RRR-A $\beta 18-27(+3)$ and DDD-A $\beta 18-27(-3)$. Therefore, the global principle ${ }^{21}$ cannot tell us which of the charged mutations leads to more favorable solvation. (This is the primary reason why this particular dAb was chosen among several related dAbs studied in Refs. ${ }^{15,16}$.) Interestingly, the solubilizing activity of RRR and DDD mutations was found to be contrastive; experimental measurements indicate that aggregation is still observable for RRR-A $\beta 18-27$, but it is eliminated for DDD-A $\beta 18-27^{16}$. Correspondingly, we find that $\Delta G_{\text {solv }}$ for DDD-A $\beta 18-27$ is strikingly more 
$\mathrm{E} 10 \mathrm{G} / \mathrm{D} 73 \mathrm{~N} / \mathrm{A} 76 \mathrm{~K}$ (heavy chain) mutant of $\mathrm{mAb} 1\left(\Delta \mathrm{G}_{\mathrm{solv}}=-343.8 \mathrm{kcal} / \mathrm{mol}\right)$
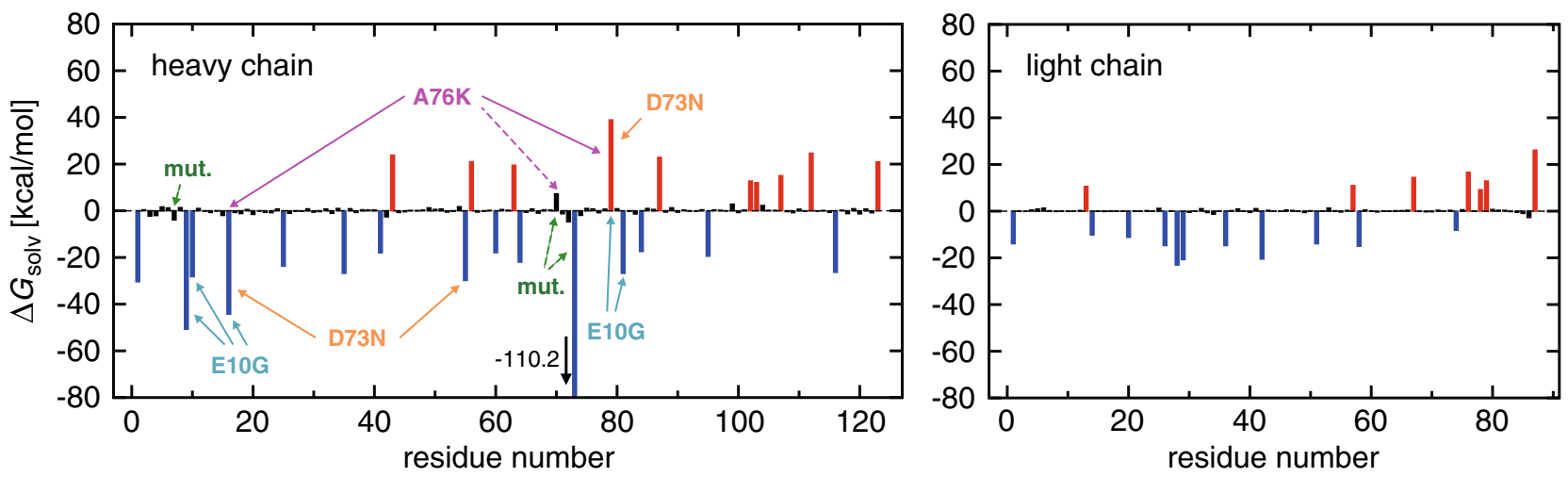

b $\mathrm{D} 60 \mathrm{~S} / \mathrm{E} 80 \mathrm{Q}$ (light chain) mutant of $\mathrm{mAb} 1\left(\Delta G_{\mathrm{solv}}=-160.9 \mathrm{kcal} / \mathrm{mol}\right)$
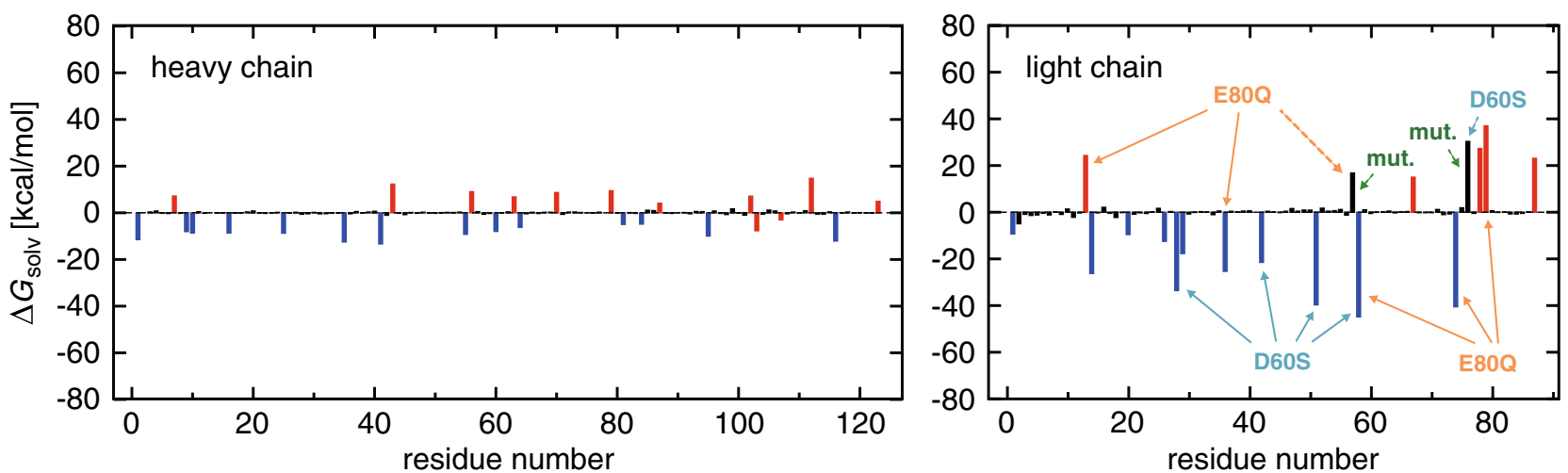

C $\mathrm{E} 10 \mathrm{G} / \mathrm{D} 73 \mathrm{~N} / \mathrm{A} 76 \mathrm{~K} / \mathrm{D} 60 \mathrm{~S} / \mathrm{E} 80 \mathrm{Q}$ (heavy and light chain) mutant of $\mathrm{mAb} 1\left(\Delta \mathrm{G}_{\text {solv }}=-503.1 \mathrm{kcal} / \mathrm{mol}\right)$
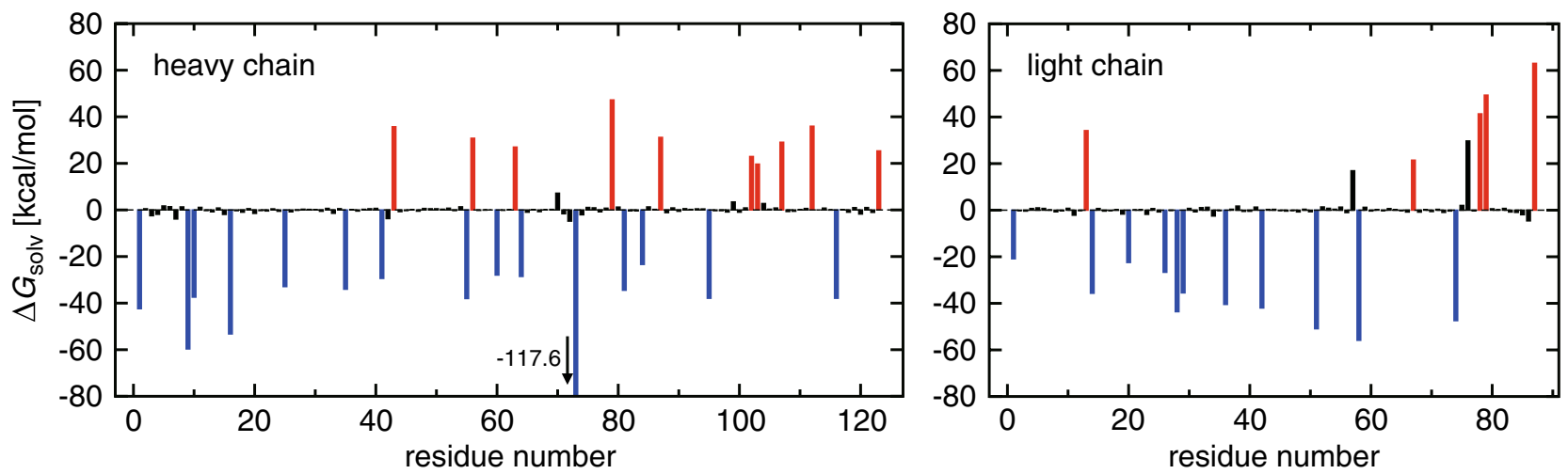

Figure 4. (a-c) Residue-wise decomposition of $\Delta G_{\text {solv }}$ for (a) E10G/D73N/A76K, (b) D60S/E80Q, and (c) E10G/D73N/A76K/D60S/E80Q mutants of mAb1 (blue, red and black colors refer to positively charged, negatively charged and neutral residues, respectively). The mutation sites are indicated by the green arrows. Charged residues that exhibit pronounced variations in individual single-point mutations (taken from Fig. 2 and Supplementary Fig. S1) are represented by the solid arrows labeled with respective mutations. When those charged residues are deleted upon multipoint mutations, the solid arrows are replaced by the dashed ones.

negative than the one for RRR-A $\beta 18-27$ (Table 3). Thus, there must be some local environment effects that distinguish positively and negatively charged residues at the N-terminus of CDR3. The residue-wise decomposition analysis of $\Delta G_{\text {solv }}$ is suited to address such local effects, and the results for RRR-A $\beta 18-27$ and DDD-A $\beta 18-27$ are shown in Fig. 6 . We first note that, since the total charge of RRR-A $\beta 18-27$ is positive, positive and negative residues respectively tend to exhibit negative and positive $\Delta G_{\text {solv }}$ values (Fig. 6a), whereas this trend is inverted for DDD-A $\beta 18$-27 (Fig. 6b). We observe from Fig. 6a,b and the insets that more negatively charged residues are present than positively charged ones within $\sim 15 \AA$ from the mutation sites. This explains why the $\Delta G_{\text {solv }}$ values at the mutation sites in RRR-A $\beta 18-27$ is much less negative than those in DDD-A $\beta 18-27$. Thus, it is the local principle, derived above from the analyses of $\mathrm{mAb} 1$ and $\mathrm{mAb} 2$, that accounts for the distinct solubilizing activity of RRR and DDD mutations. 

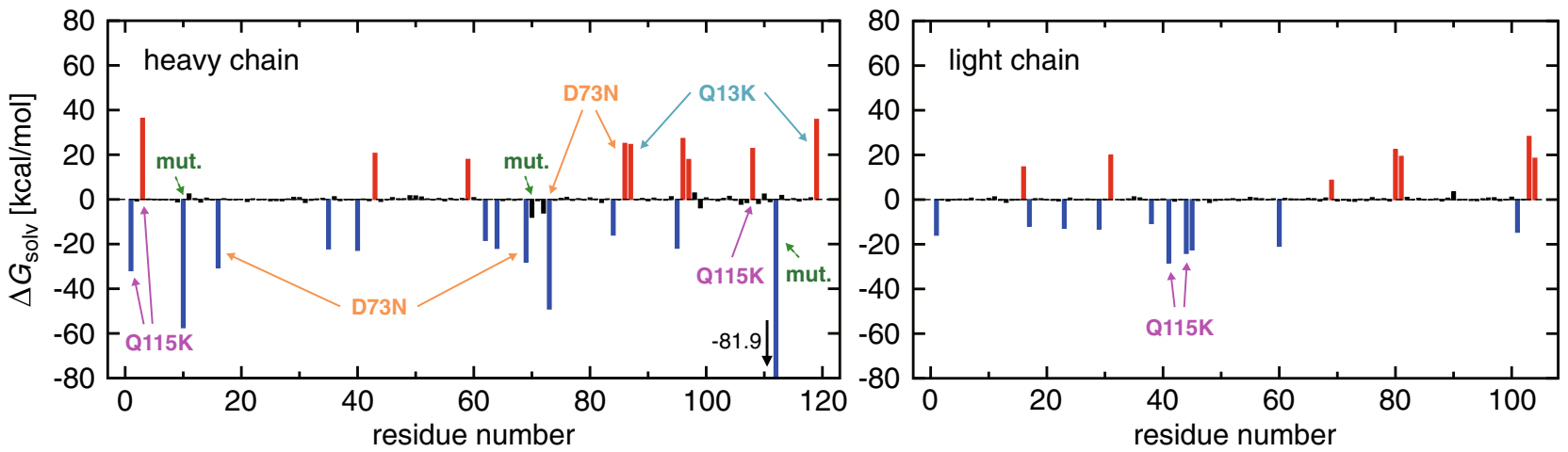

b D32Y/S77R (light chain) mutant of $\mathrm{mAb2}\left(\Delta G_{\mathrm{solv}}=-12.2 \mathrm{kcal} / \mathrm{mol}\right)$
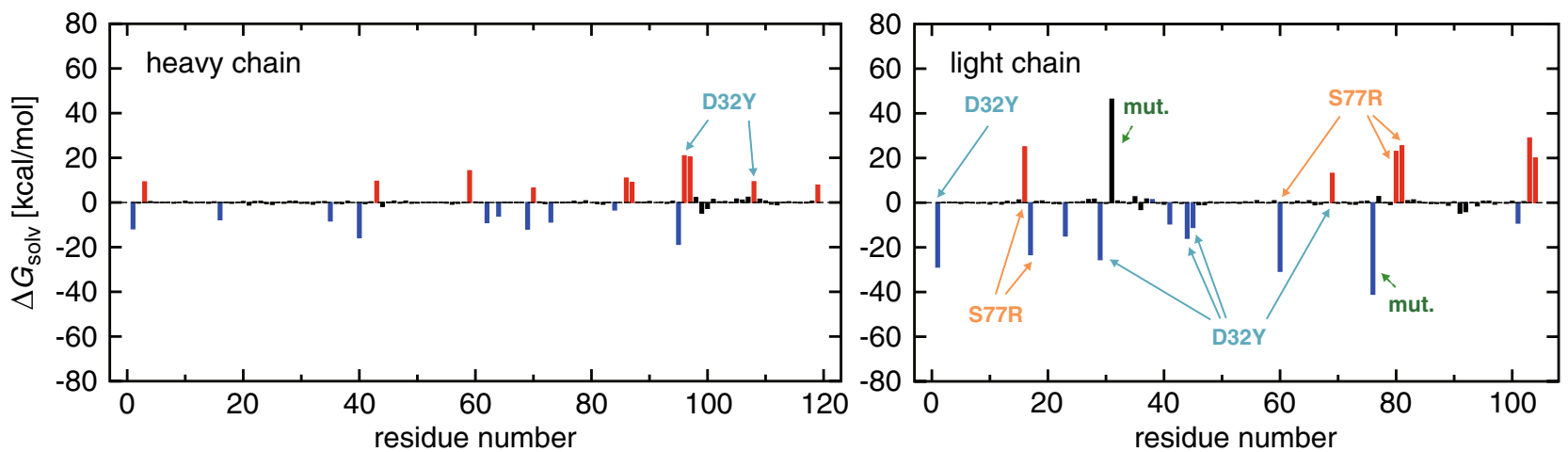

C Q13K/D73N/Q115K/D32Y/S77R (heavy and light chain) mutant of $m A b 2\left(\Delta G_{\text {solv }}=-316.1 \mathrm{kcal} / \mathrm{mol}\right)$
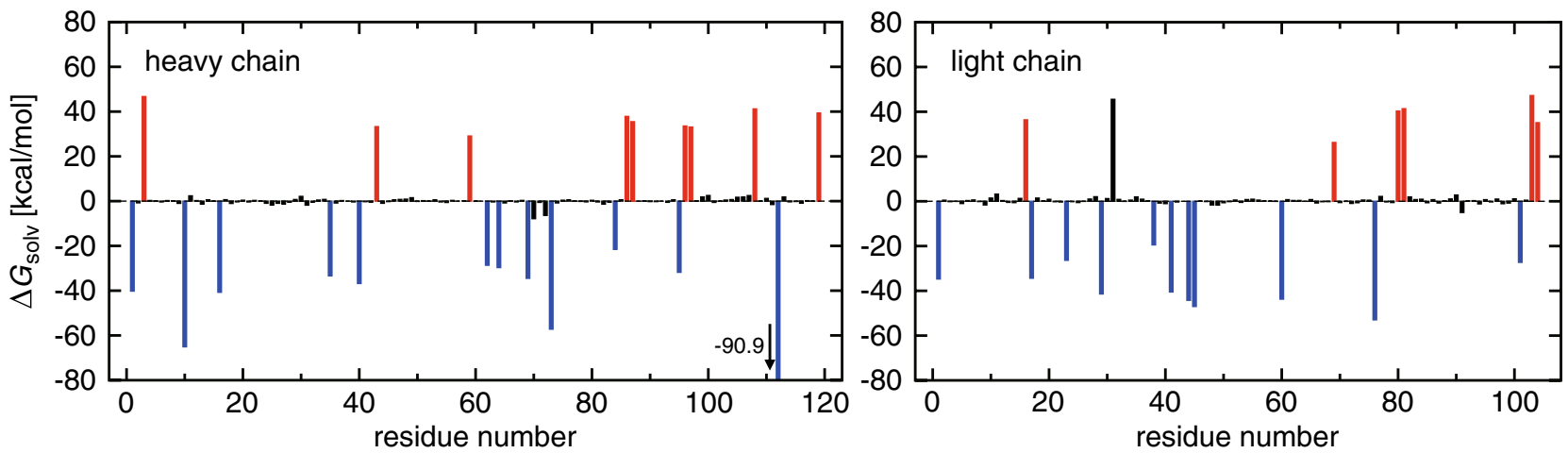

Figure 5. (a-c) Residue-wise decomposition of $\Delta G_{\text {solv }}$ for (a) Q13K/D73N/Q115K, (b) D32Y/S77R, and (c) Q13K/D73N/Q115K/D32Y/S77R mutants of mAb2 (blue, red and black colors refer to positively charged, negatively charged and neutral residues, respectively). The mutation sites are indicated by the green arrows. Charged residues that exhibit pronounced variations in individual single-point mutations (taken from Fig. 3 and Supplementary Fig. S2) are represented by the solid arrows labeled with respective mutations.

\section{Discussion}

Protein aggregation has been an area of major focus in its own right because of its relation to human diseases ${ }^{41}$. Factors promoting aggregation-prone nature have therefore been intensively investigated, and several algorithms have come out to rationalize and predict protein aggregation propensity ${ }^{42-45}$. These algorithms mainly focus on sequence characteristics such as the $\beta$-sheet forming propensity and the amino acid hydrophobicity. They have also been applied to identify aggregation-prone regions in antibodies, and it was found that such regions are typically located within CDRs responsible for antigen binding ${ }^{46-48}$. On the other hand, we have recently proposed a different type of strategy from a solvation viewpoint ${ }^{21}$. This is natural since whether a protein becomes aggregation-prone or remains soluble in aqueous environments should critically depend on the affinity for water. In fact, it was demonstrated that the solvation free energy is the key factor controlling the protein aggregation propensity ${ }^{21}$. Since the solvation free energy is largely affected by charged residues, we could thereby derive a design principle for increasing the resistance to aggregation through charged mutations. 
a
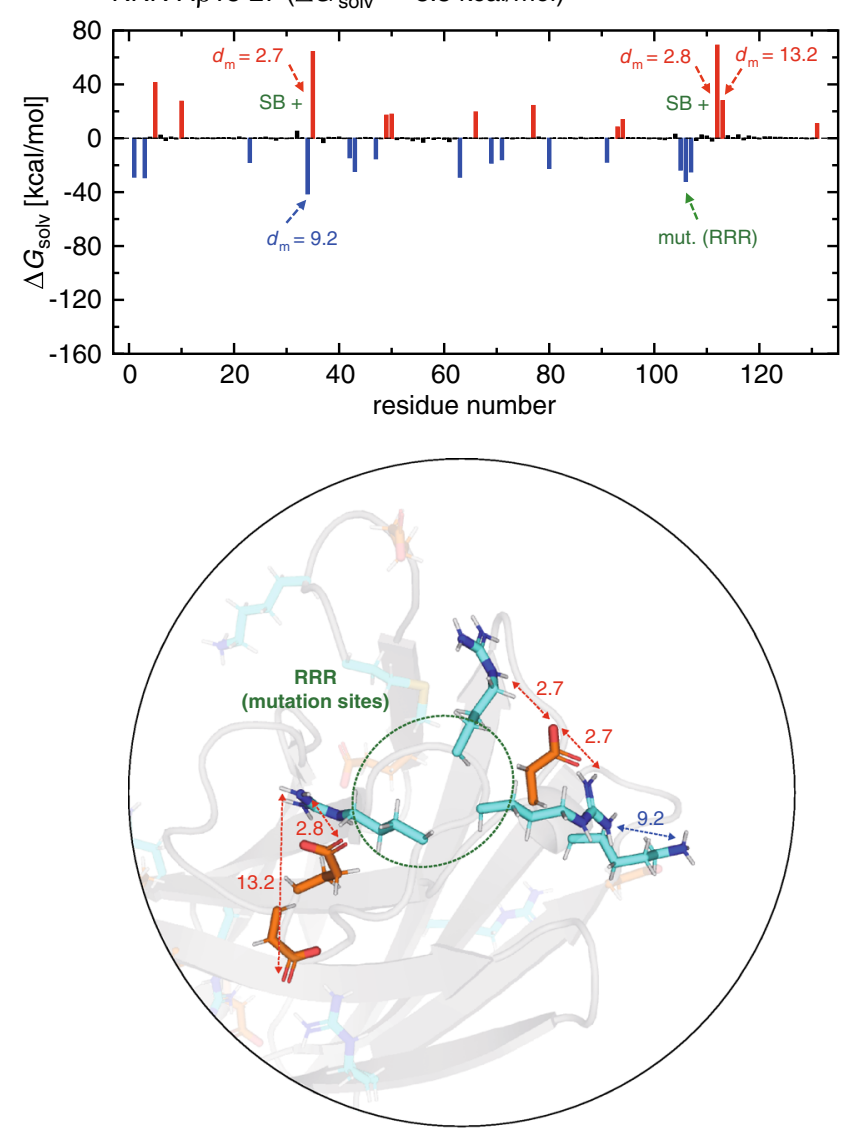

b

$\operatorname{DDD}-\mathrm{A} \beta 18-27\left(\Delta G_{\text {solv }}=-507.7 \mathrm{kcal} / \mathrm{mol}\right)$
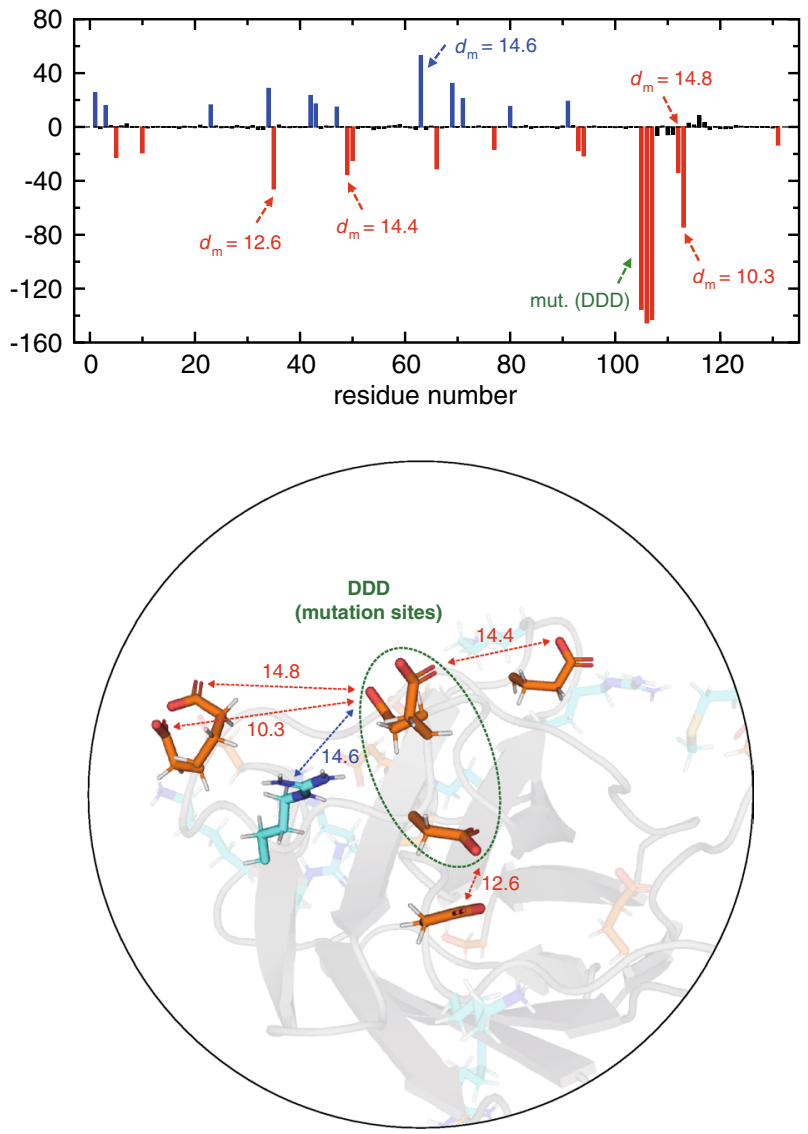

Figure 6. (a, b) Residue-wise decomposition of $\Delta G_{\text {solv }}$ for (a) RRR-A $\beta 18-27$ and (b) DDD-A $\beta 18-27$ mutants of $\mathrm{A} \beta 18-27 \mathrm{dAb}$ (blue, red and black colors refer to positively charged, negatively charged and neutral residues, respectively). The mutation sites are indicated by the green arrow. $d_{\mathrm{m}}$ (in $\AA$ ) denotes the distance to the mutation sites as illustrated in the inset (green dashed oval, mutation sites; cyan and orange stick representations, positively and negatively charged residues, respectively). Residues involved in the formation/breaking of salt-bridges upon mutation are represented by $\mathrm{SB}+/$ - (green if the mutation sites are involved, and black otherwise). PyMOL version 1.8.2 (https://pymol.org) was used to generate protein figures.

However, the previously derived principle is a global one in the sense that it takes into consideration only the global net charge of a protein and the sign (positive or negative) of charged mutations. It therefore cannot discriminate, e.g., the Q13K and Q115K mutants of mAb2 studied in the present work, which however exhibit strikingly contrasting solvation free energy changes $(+12.0$ and $-43.6 \mathrm{kcal} / \mathrm{mol}$, respectively; see Table 2$)$. Through the residue-wise decomposition analysis of the solvation free energy changes, it is elucidated that the contrasting behavior of the Q13K and Q115K mutants is caused by the difference in local charge surrounding the mutation site. Thus, the spatial distribution of nearby ( $<\sim 15 \AA)$ charged residues is found to significantly impact the solvation free energy change. We also observe that the effects of multipoint mutations can be roughly described as a superposition of those from single-point mutations. Furthermore, our analysis for mAb2 reveals why multiple mutations (more than two) are sometimes necessary to confer sufficiently negative solvation free energy. These results are in accord with the experimental observation that multiple mutations are necessary to considerably improve aggregation resistance and their effects are largely additive ${ }^{17}$.

Antibodies of neutral net charge are also the systems for which the previously derived global principle alone is insufficient: it cannot propose which type (positive or negative) of charged mutations should be introduced to enhance the solubility. In this connection, it was observed experimentally that inserting negatively charged residues (DDD) is more beneficial than inserting positively charged residues (RRR) in preventing the aggregation of $\mathrm{A} \beta 18-27 \mathrm{dAb}$ of neutral net charge ${ }^{16}$. This observation cannot be rationalized by the electrostatic repulsion since its magnitude is the same between positive charges and between negative charges. On the other hand, we demonstrate that the solvation free energy clearly discriminates DDD-A $\beta 18-27$ and RRR-A $\beta 18-27$ and that it can be accounted for by the local environment surrounding the mutation sites. This indicates a potential advantage of adopting the solvation viewpoint in arguing protein aggregation.

Finally, let us make comments related to the convergence of solvation free energy calculations. As stated at the beginning of Results section, additional two independent $100 \mathrm{~ns}$ simulations were carried out for the Q13K, S77R and D32Y/S77R mutants of $\mathrm{mAb} 2$, and non-negligible differences were found between the average $\Delta G_{\text {solv }}$ values computed from the original and additional simulations (Supplementary Table S3). To examine 
the discrepancies, we plotted $\Delta G_{\text {solv }}$ as a function of simulation time for individual trajectories (Supplementary Figs. S4, S5 and S6). We observe that $\Delta G_{\text {solv }}$ values fluctuate significantly (the standard deviation in individual trajectories is $\sim 70$ to $90 \mathrm{kcal} / \mathrm{mol}$ ). Such large fluctuations in individual trajectories reflect the fact that the solvation free energy depends on fine details of the underlying protein structure: for example, the formation of just a single salt-bridge in a protein can lead to $\sim 50 \mathrm{kcal} / \mathrm{mol}$ variation ${ }^{23}$. Therefore, even subtle differences in the population of hydrogen bonds and salt-bridges in different trajectories can significantly affect the average $\Delta G_{\text {solv }}$ value, and it is extremely difficult to obtain its converged result (e.g., within an error of $\sim 1 \mathrm{kcal} / \mathrm{mol}$ ). The average $\Delta G_{\text {solv }}$ values reported in the present work were computed from relatively short (100 ns) simulations, with which typical errors are of the order of $\sim 10 \mathrm{kcal} / \mathrm{mol}$ (Tables 1,2 and 3). Hence, the average $\Delta G_{\text {solv }}$ values for the Q13K, S77R and D32Y/S77R mutants of mAb2 are not decisive from our calculations: $\Delta G_{\text {solv }} \sim \pm 10$ $\mathrm{kcal} / \mathrm{mol}$ for these mutants with errors of the order of $\sim 10 \mathrm{kcal} / \mathrm{mol}$.

Nevertheless, this observation does not invalidate the present work. First, the global and local effects on $\Delta G_{\text {solv }}$ upon charged mutation - the main points of our work-are not affected. This is also demonstrated in Supplementary Figs. S4, S5 and S6, where the residue-wise decompositions of $\Delta G_{\text {solv }}$ computed from the original and additional simulations are compared. We observe that the global effect is conserved: positive (negative) residues tend to exhibit negative (positive) solvation free energy changes in both the the original and additional simulations, and this can be explained by the fact that the net charge of the mutants under consideration is positive. In addition, the same local effects are observed in both the the original and additional simulations: the charged residues within $\sim 15 \AA$ from the mutation site(s) are affected in the same manner. Second, our goal is to provide design principles of aggregation-resistant antibodies upon charged mutations. As stated above, it is already recognized that multiple charged mutations are necessary to improve the aggregation resistance ${ }^{17}$. For the multiple charged mutations, the typical magnitude of $\Delta G_{\text {solv }}$ significantly exceeds $100 \mathrm{kcal} / \mathrm{mol}$ (see Tables 1,2 and 3), which is reliable even with errors of $\sim 10 \mathrm{kcal} / \mathrm{mol}$. Thus, although there is a problem if our work is concerned with the precise prediction of $\Delta G_{\text {solv }}$, the convergence of the global and local effects is attained already with the simulations reported here and our computational methods are reliably applicable in designing aggregationresistant antibodies. (Mutants for which $\Delta G_{\text {solv }} \sim \pm 10 \mathrm{kcal} / \mathrm{mol}$, such as Q13K, S77R and D32Y/S77R mentioned above, can simply be excluded from candidates of aggregation-resistant antibodies).

\section{Conclusions}

In summary, we explore here strategies for designing aggregation-resistant antibodies by charged mutations. This is done on the basis of a solvation thermodynamics perspective rather than focusing on the sequence characteristics. By combining our previous work and the analyses presented here, we identify three critical factors that significantly impact charged mutations: (1) the total net charge of an antibody; (2) the contents of mutation (the choice of the positively or negatively charged residues and avoiding the use of residues of bulky side chains); and (3) the local net charge within $\sim 15 \AA$ from the mutation site. The third factor accounts for the location dependence of charged mutations, and needs to be taken into consideration in searching for an optimal insertion location. A prominent advantage of charged mutations is that they can be inserted outside the complementarity-determining regions mediating target binding, i.e., the antibody solubility can in principle be improved without reducing the binding affinity. We hope that our design principles will be useful in producing aggregation-resistant antibody therapeutics.

Received: 9 May 2020; Accepted: 11 November 2020

Published online: 03 December 2020

\section{References}

1. Nelson, A. L., Dhimolea, E. \& Reichert, J. M. Development trends for human monoclonal antibody therapeutics. Nat. Rev. Drug Discov. 9, 767-774 (2010).

2. Sliwkowski, M. X. \& Mellman, I. Antibody therapeutics in cancer. Science 341, 1192-1198 (2013).

3. Carter, P. J. \& Lazar, G. A. Next generation antibody drugs: Pursuit of the high-hanging fruit. Nat. Rev. Drug Discov. 17, 197-223 (2018).

4. Lowe, D. et al. Aggregation, stability, and formulation of human antibody therapeutics. Adv. Protein Chem. Struct. Biol. 84, 41-61 (2011).

5. Li, W. et al. Antibody aggregation: Insights from sequence and structure. Antibodies 5, 19 (2016).

6. Jespers, L., Schon, O., Famm, K. \& Winter, G. Aggregation-resistant domain antibodies selected on phage by heat denaturation. Nat. Biotechnol. 22, 1161-1165 (2004).

7. Holliger, P. \& Hudson, P. J. Engineered antibody fragments and the rise of single domains. Nat. Biotechnol. 23, 1126-1136 (2005).

8. Lee, C. C., Perchiacca, J. M. \& Tessier, P. M. Toward aggregation-resistant antibodies by design. Trends Biotechnol. 31, 612-620 (2013).

9. Roberts, C. J. Therapeutic protein aggregation: Mechanisms, design and control. Trends Biotechnol. 32, 372-380 (2014).

10. Lawrence, M. S., Phillips, K. J. \& Liu, D. R. Supercharging proteins can impart unusual resilience. J. Am. Chem. Soc. 129, 1011010112 (2007).

11. Wu, S.-J. et al. Structure-based engineering of a monoclonal antibody for improved solubility. Protein Eng. Des. Sel. 23, 643-651 (2010).

12. Simeonov, P., Berger-Hoffmann, R., Hoffmann, R., Sträter, N. \& Zuchner, T. Surface supercharged human enteropeptidase light chain shows improved solubility and refolding yield. Protein Eng. Des. Sel. 24, 261-268 (2011).

13. Perchiacca, J. M., Bhattacharya, M. \& Tessier, P. M. Mutational analysis of domain antibodies reveals aggregation hotspots within and near the complementarity determining regions. Proteins 79, 2637-2647 (2011).

14. Miklos, A. E. et al. Structure-based design of supercharged, highly thermoresistant antibodies. Chem. Biol. 19, 449-455 (2012).

15. Perchiacca, J. M., Ladiwala, A. R. A., Bhattacharya, M. \& Tessier, P. M. Aggregation-resistant domain antibodies engineered with charged mutations near the edges of the complementarity-determining regions. Protein Eng. Des. Sel. 25, 591-601 (2012). 
16. Perchiacca, J. M., Lee, C. C. \& Tessier, P. M. Optimal charged mutations in the complementarity-determining regions that prevent domain antibody aggregation are dependent on the antibody scaffold. Protein Eng. Des. Sel. 27, 29-39 (2014).

17. Dudgeon, K. et al. General strategy for the generation of human antibody variable domains with increased aggregation resistance. Proc. Natl. Acad. Sci. USA 109, 10879-10884 (2012).

18. Kauzmann, W. Some factors in the interpretation of protein denaturation. Adv. Protein. Chem. 14, 1-64 (1959).

19. Tanford, C. The hydrophobic effect and the organization of living matter. Science 200, 1012-1018 (1978).

20. Ben-Naim, A. Hydrophobic Interactions (Plenum Press, New York, 1980).

21. Chong, S.-H. \& Ham, S. Interaction with the surrounding water plays a key role in determining the aggregation propensity of proteins. Angew. Chem. Int. Ed. 53, 3961-3964 (2014).

22. Kuhn, A. B. et al. Improved solution-state properties of monoclonal antibodies by targeted mutations. J. Phys. Chem. B 121, 10818-10827 (2017).

23. Chong, S.-H. \& Ham, S. Atomic decomposition of the protein solvation free energy and its application to amyloid-beta protein in water. I. Chem. Phys. 135, 034506 (2011).

24. Chong, S.-H. \& Ham, S. Site-directed analysis on protein hydrophobicity. J. Comput. Chem. 35, 1364-1370 (2014).

25. Chong, S.-H. \& Ham, S. Distinct role of hydration water in protein misfolding and aggregation revealed by fluctuating thermodynamics analysis. Acc. Chem. Res. 48, 956-965 (2015).

26. Burton, D. R. et al. Efficient neutralization of primary isolates of HIV-1 by a recombinant human monoclonal antibody. Science 266, 1024-1027 (1994)

27. Rioux, J. D., Zdárský, E., Newkirk, M. M. \& Rauch, J. Anti-DNA and anti-platelet specificities of SLE-derived autoantibodies: Evidence for $\mathrm{CDR} 2_{\mathrm{h}}$ mutations and CDR $3_{\mathrm{h}}$ motifs. Mol. Immunol. 32, 683-696 (1995).

28. Azoitei, M. L. et al. Computation-guided backbone grafting of a discontinuous motif onto a protein scaffold. Science 334, 373-376 (2011).

29. Maier, J. K. \& Labute, P. Assessment of fully automated antibody homology modeling protocols in molecular operating environment. Proteins 82, 1599-1610 (2014).

30. He, X. M., Rüker, F., Casale, E. \& Carter, D. C. Structure of a human monoclonal antibody Fab fragment against gp41 of human immunodeficiency virus type 1. Proc. Natl. Acad. Sci. USA 89, 7154-7158 (1992).

31. Perchiacca, J. M., Ladiwala, A. R. A., Bhattacharya, M. \& Tessier, P. M. Structure-based design of conformation- and sequencespecific antibodies against amyloid $\beta$. Proc. Natl. Acad. Sci. USA 109, 84-89 (2012).

32. Barthelemy, P. A. et al. Comprehensive analysis of the factors contributing to the stability and solubility of autonomous human VH domains. J. Biol. Chem. 283, 3639-3654 (2008).

33. Case, D. A. et al. AMBER 18 (University of California, San Francisco, 2018).

34. Maier, J. A. et al. ff14SB: Improving the accuracy of protein side chain and backbone parameters from ff99SB. J. Chem. Theory Comput. 11, 3696-3713 (2015).

35. Jorgensen, W. L., Chandrasekhar, J., Madura, J. D., Impey, R. W. \& Klein, M. L. Comparison of simple potential functions for simulating liquid water. J. Chem. Phys. 79, 926-935 (1983).

36. Darden, T., York, D. \& Pedersen, L. Particle mesh Ewald: An $n$ - $\log (n)$ method for Ewald sums in large systems. J. Chem. Phys. 98, 10089-10092 (1993).

37. Berendsen, H. J. C., Postma, J. P. M., van Gunsteren, W. F., DiNola, A. \& Haak, J. R. Molecular dynamics with coupling to an external bath. J. Chem. Phys. 81, 3684-3690 (1984).

38. Kovalenko, A. Three-dimensional RISM theory for molecular liquids and solid-solid interfaces. In Molecular Theory of Solvation (ed. Hirata, F.) 169-275 (Kluwer Academic, Dordrecht, 2003).

39. Imai, T., Harano, Y., Kinoshita, M., Kovalenko, A. \& Hirata, F. A theoretical analysis on hydration thermodynamics of proteins. J. Chem. Phys. 125, 024911 (2006).

40. Atkins, P. \& de Paula, J. Physical Chemistry 8th edn. (Oxford University Press, Oxford, 2006).

41. Chiti, F. \& Dobson, C. M. Protein misfolding, functional amyloid, and human disease. Annu. Rev. Biochem. 75, 333-366 (2006).

42. Chiti, F., Stefani, M., Taddei, N., Ramponi, G. \& Dobson, C. M. Rationalization of the effects of mutations on peptide and protein aggregation rates. Nature 424, 805-808 (2003).

43. Fernandez-Escamilla, A.-M., Rousseau, F., Schymkowitz, J. \& Serrano, L. Prediction of sequence-dependent and mutational effects on the aggregation of peptides and proteins. Nat. Biotechnol. 22, 1302-1306 (2004).

44. Tartaglia, G. G. \& Vendruscolo, M. The zyggregator method for predicting protein aggregation propensities. Chem. Soc. Rev. 37, 1395-1401 (2008).

45. Maurer-Stroh, S. et al. Exploring the sequence determinants of amyloid structure using position-specific scoring matrices. Nat. Methods 7, 237-242 (2010).

46. Wang, X., Das, T. K., Singh, S. K. \& Kumar, S. Potential aggregation prone regions in biotherapeutics: A survey of commercial monoclonal antibodies. mAbs 1, 254-267 (2009).

47. Wang, X., Singh, S. K. \& Kumar, S. Potential aggregation-prone regions in complementarity-determining regions of antibodies and their contribution towards antigen recognition: A computational analysis. Pharm. Res. 27, 1512-1529 (2010).

48. Buck, P. M. et al. Computational methods to predict therapeutic protein aggregation. Methods Mol. Biol. 899, 425-451 (2012).

\section{Acknowledgements}

This work was supported by the Samsung Science and Technology Foundation under Project Number SSTF-BA1401-52 and the National Research Foundation of Korea (NRF) (NRF-2017R1A2B3010053 and NRF-2018R1D1A1B07045862).

\section{Author contributions}

J.L., S.-H.C. and S.H. conducted the research and wrote the manuscript.

\section{Competing interests}

The authors declare no competing interests.

\section{Additional information}

Supplementary information is available for this paper at https://doi.org/10.1038/s41598-020-78136-1.

Correspondence and requests for materials should be addressed to S.H.

Reprints and permissions information is available at www.nature.com/reprints.

Publisher's note Springer Nature remains neutral with regard to jurisdictional claims in published maps and institutional affiliations. 
(c) (i) Open Access This article is licensed under a Creative Commons Attribution 4.0 International cc) License, which permits use, sharing, adaptation, distribution and reproduction in any medium or format, as long as you give appropriate credit to the original author(s) and the source, provide a link to the Creative Commons licence, and indicate if changes were made. The images or other third party material in this article are included in the article's Creative Commons licence, unless indicated otherwise in a credit line to the material. If material is not included in the article's Creative Commons licence and your intended use is not permitted by statutory regulation or exceeds the permitted use, you will need to obtain permission directly from the copyright holder. To view a copy of this licence, visit http://creativecommons.org/licenses/by/4.0/.

(C) The Author(s) 2020 